\title{
Managing UTI in primary care: should we be sending midstream urine samples?
}

The decision to prescribe antibiotics is one of the most common treatment decisions faced by frontline primary care clinicians daily, and urinary tract infection (UTI) is one of the most common bacterial infections encountered. ${ }^{1} \quad$ Although antimicrobial treatment for UTI is accepted in clinical practice, antibiotic resistance in urinary bacteria is increasing, ${ }^{2}$ with rates cited in the literature of between $20 \%$ and $40 \%$ to trimethoprim and amoxicillin respectively. ${ }^{3}$ Bacteria are adept at side-stepping human intervention (for example, antibiotics and vaccines) and are developing resistance to antibiotics faster than the pharmaceutical industry is developing new ones: only two new classes of antibiotics have been developed in the last 30 years. ${ }^{4}$

These problems highlight the need for high quality evidence to help primary care clinicians optimise the diagnosis and management of UTI. Fortunately, a plethora of high quality, clinically valuable, primary care research papers have been published recently, including four in the $B M J^{5-8}$ and two in this month's BJGP. ${ }^{9,10}$

One of the BMJ papers reported that a range of management strategies (immediate versus delayed empirical antibiotics versus antibiotics guided by the results of dipstick versus symptom score versus midstream urine culture) achieved similar symptomatic results, while delayed empirical and dipstick-guided prescribing reduced antibiotic consumption. ${ }^{5}$ The authors concluded that there is "no advantage in routinely sending midstream urine [MSU] samples for [culture]'. This makes for an interesting and contrasting background to one of the papers reported in this month's BJGP, ${ }^{9}$ which provides evidence in support of sending MSUs for culture.

This study by Vellinga et al provides some original and novel evidence for a practice, I suspect, is commonly used in primary care: that of looking for a previous urine culture to guide the treatment of a current, suspected UTI. Although it has made intuitive sense to avoid an antibiotic to which a patient's urinary bacteria have previously been found to be resistant, until now there has been no evidence to support this practice. Furthermore, when it was used, clinicians must have wondered how long previous results remain relevant and if resistance to some antibiotics is more important than others.

The study by Vellinga and colleagues addresses both these questions. They found that a previous measure of resistance to co-amoxiclav, ampicillin, ciprofloxacin, and trimethoprim remains predictive of further resistance at 3 and 12 months. The long duration of effects, although not as strong as at 3 months, may surprise many clinicians, but is not an isolated finding. A recently published systematic review ${ }^{11}$ reports a similar trend of effects in three studies ${ }^{3,12,13}$ that, when pooled, show that the relationship between antibiotics prescribed over a 12-month period and bacterial resistance cannot be explained by chance (odds ratio $=1.33$, $95 \% \mathrm{Cl}=1.15$ to 1.53 ).

Happily, their findings do not contradict the Health Protection Agency's antibiotic prescribing guidance ${ }^{14}$ that nitrofurantoin should be used as a first line treatment. In the study dataset, this was the antibiotic to which fewest bacteria were resistant at baseline, 3 and 12 months; and the antibiotic with the lowest positive and highest negative predictive values. In other words, previous resistance was least likely to predict future resistance, and previous susceptibility was most likely to predict future susceptibility.

I think there are two potential study limitations of Vellinga et al's study ${ }^{9}$ which we should be aware of. First, the need to distinguish between a recurrent infection versus detecting the same infection twice. The authors' chose a threshold of 2 weeks; that is, if a patient was found to have a repeat infection more than 2 weeks after the index infection, it was deemed a recurrence. This is a classic point of uncertainty for any study design, as there will be little, if any, evidence available to assist decision making and whatever is selected could be criticised as being too generous or lenient. However, given that one trial showed that $88 \%$ of women with UTI treated with nitrofurantoin had both improved and were microbiologically cured at 7 days, ${ }^{15} 2$ weeks does not seem unreasonable. Second, it would help us interpret the generalisability (otherwise known as the external validity or the extent to which the study patients are similar to our patients) if we know how Galway GPs in the Vellinga et al study use urine sampling. For example, is the local policy to send samples on all patients with suspected UTI, or only those with atypical symptoms, or patients deemed to be at high risk?

The second study ${ }^{10}$ in this issue of the $B J G P$ makes an important contribution to improving our understanding of the relative contribution that symptoms, signs, and near-patient tests make to the diagnosis and prognosis of infections, or put more simply, developing diagnostic/prognostic algorithms. There are three stages in the development of such algorithms. First derivation, in which candidate symptoms, signs, or near-patient test results (the 'predictors') are identified as being statistically associated with the diagnosis (or prognosis) in question. Second validation, where predictors are checked in a different dataset to see if they remain associated, and to a similar degree. Finally, the use of the validated algorithm should be compared to usual care in a randomised controlled trial to see if patient outcomes are improved.

The Little et al study ${ }^{10}$ is an example of a second (validation) stage study, of which there are surprisingly few in primary care. It addresses the issue of accurately diagnosing UTI in women, which is vital if we are to target antibiotics at those who are most likely to benefit (and withhold them from those who are unlikely to). And the diagnosis is by no means clinically straightforward with only $50 \%$ to $66 \%$ of women with suspected UTI having a 
microbiologically confirmed infection. ${ }^{10,16}$ The Little et al study is well designed and executed, and largely confirms the findings of its sister derivation study, ${ }^{17}$ showing that dipstick urinalysis contributes more to diagnosis than symptoms. The investigators found that the presence of nitrites or both leucocytes plus blood raised the probability of UTI from 0.66 (pretest probability) to 0.81 (post-test probability, otherwise known as the positive predictive value). If nitrites and either blood or leucocytes were present, the post-test probability was raised further to 0.92. However, the absence of any of nitrite, leucocytes, or blood did not adequately rule out UTI, and even when all three were absent the probability of UTI was still 0.24 (negative predictive value was 0.76).

Examining the study's limitations, first the investigators had a tricky choice regarding the laboratory threshold for the diagnosis of UTI: do they 'future and Euro proof it', by using the European accepted definition of $>10^{3} \mathrm{cfu} / \mathrm{ml}$ (colony-forming units) or go with the UK/NHS standard of $>10^{5} \mathrm{cfu} / \mathrm{ml}$. They chose the lower threshold, so it is possible that associations between symptoms, signs, and dipstick urinalysis results may differ in this study to daily clinical practice. This may, in part, be responsible for the relatively poor negative predictive values since, if the diagnostic threshold for UTI is lower and more UTIs are being diagnosed, it is harder to rule it out.

Second, although it is not explicitly stated in the eligibility criteria, the study appears to have excluded women with upper UTI (perhaps those with more severe systemic symptoms and loin pain). Most women with lower urinary tract symptoms are not routinely examined for supra-pubic or loin tenderness in primary care. This makes the inclusion of the measurement of these signs more challenging for researchers, as they would be asking clinicians to conduct an additional examination in a busy, time pressured, emergency clinic. However, it also means that the study cannot assess the diagnostic value (positive or negative prediction) of these signs.

In summary, the evidence reviewed suggests a role for urine dipstick in ruling in
$\mathrm{UTI}{ }^{10}$ and reducing antibiotic use. ${ }^{5}$ What is less clear is whether we should routinely send MSUs. If we stop, we could save money, ${ }^{6}$ but as Srirangam and Bondin describe, ${ }^{18}$ we could be losing in three ways. First, urine cultures provide valuable information regarding the changing patterns of antimicrobial resistance (a societal benefit). Second, as shown by Vellinga et $a l,{ }^{9}$ a proportion of patients experience repeated UTIs, and previous results can help guide subsequent treatment (a patient benefit). Third, as discussed, between $34 \%$ and $50 \%$ of women presenting with urinary symptoms do not have a UTI, and we currently do not have any other sufficiently accurate diagnostic test to detect these patients. To these three I would add a fourth, namely clinical curiosity. Positive and negative predictive values only estimate group average probabilities of UTI, but the only way of establishing the diagnosis for the individual patient, with their unique history and set of circumstances, is a urine culture.

It is surely the case that 'not all UTIs are the same' and that there may be some patients in whom MSU is not cost-effective (from either a patient or societal perspective), but that in others (perhaps older patients, those with more severe symptoms, or those with recurrent UTI) there is a role for MSU. Further research is needed.

\section{Alastair D Hay,}

Consultant Senior Lecturer in Primary Health Care, Academic Unit of Primary Health Care, NIHR National School for Primary Care Research, Department of Community Based Medicine, University of Bristol.

\section{Provenance}

Commissioned; not peer reviewed.

\section{REFERENCES}

1. McCormick A, Fleming D, Charlton J. Morbidity statistics from general practice - fourth national study 1991-1992. London: HMSO, 1995.

2. Department for Environment FaRA. Overview of antimicrobial usage and bacterial resistance in selected human and animal pathogens in the UK: 2007. Department of Health, 2010.

3. Hillier S, Roberts Z, Dunstan F, et al. Prior antibiotics and risk of antibiotic-resistant community-acquired urinary tract infection: a case-control study. $J$ Antimicrob Chemother 2007; 60(1): 92-99.

4. Infectious Diseases Society of America. Bad bugs, no drugs: as antibiotic discovery stagnate and a public health crisis brews. Arlington, VA, US: Infectious Diseases Society of America, 2004.
5. Little P, Moore MV, Turner S, et al. Effectiveness of five different approaches in management of urinary tract infection: randomised controlled trial. BMJ 2010; 340: c199.

6. Turner D, Little P, Raftery J, et al. Cost effectiveness of management strategies for urinary tract infections: results from randomised controlled trial. BMJ 2010; 340: c346.

7. Leydon GM, Turner S, Smith H, Little P, on behalf of the UTIS team. Women's views about management and cause of urinary tract infection: qualitative interview study. BMJ 2010; 340: c279.

8. Little P, Merriman R, Turner S, et al. Presentation, pattern, and natural course of severe symptoms, and role of antibiotics and antibiotic resistance among patients presenting with suspected uncomplicated urinary tract infection in primary care: observational study. BMJ 2010; 340: b5633.

9. Vellinga A, Cormican M, Hanahoe B, Murphy AW. Predictive value of antimicrobial susceptibility from previous urinary tract infection in the treatment of reinfection. Br J Gen Pract 2010; 60: 511-513.

10. Little P, Turner S, Rumsby K, et al. Validating the prediction of lower urinary tract infection in primary care: sensitivity and specificity of urinary dipsticks and clinical scores in women. Br J Gen Pract 2010; 60 495-500.

11. Costelloe C, Metcalfe C, Lovering A, et al. Effect of antibiotic prescribing in primary care on antimicrobial resistance in individual patients: systematic review and meta-analysis. BMJ 2010; 340: c2096.

12. Donnan PT, Wei L, Steinke DT, et al. Presence of bacteriuria caused by trimethoprim resistant bacteria in patients prescribed antibiotics: multilevel model with practice and individual patient data. BMJ 2004; 328(7451): 1297.

13. Hay AD, Thomas M, Montgomery A, et al. The relationship between primary care antibiotic prescribing and bacterial resistance in adults in the community: a controlled observational study using individual patient data. J Antimicrob Chemother 2005; 56(1): 146-153.

14. Health Protection Agency. Management of Infection Guidance for Primary Care for Consultation and Local Adaptation. London: Health Protection Agency, 2010.

15. Christiaens TC, De Meyere M, Verschraegen G, et al. Randomised controlled trial of nitrofurantoin versus placebo in the treatment of uncomplicated urinary tract infection in adult women. Br J Gen Pract 2002; 52(482): 729-734.

16. Bent S, Nallamothu BK, Simel DL, et al. Does this woman have an acute uncomplicated urinary tract infection? JAMA 2002; 287(20): 2701-2710.

17. Little P, Turner S, Rumsby K, et al. Developing clinical rules to predict urinary tract infection in primary care settings: sensitivity and specificity of near patient tests (dipsticks) and clinical scores. Br J Gen Pract 2006; 56(529): 606-612.

18. Srirangam SJ, Bondin D. UTI in primary care. Midstream urine sampling is still helpful. [Letter] BMJ 2010; 340: c1859.

DOI: 10.3399/bjgp10X514701

ADDRESS FOR CORRESPONDENCE

\section{Dr Alastair Hay}

Academic Unit of Primary Health Care, NIHR National School for Primary Care Research, Department of Community Based Medicine, University of Bristol, 25 Belgrave Road, Bristol BS8 2AA. E-mail: alastair.hay@bristol.ac.uk 